\title{
Human stem cell-derived cardiac model of chronic drug exposure
}

Sara S. Nunes, Nicole Feric, Aric Pahnke, Jason W. Miklas, Mark Li, John Coles, Mark Gagliardi, Gordon

Keller and Milica Radisic

Number of pages including cover: 5

Figures: 3

Tables:1 


\section{Supplemental Figure 1}

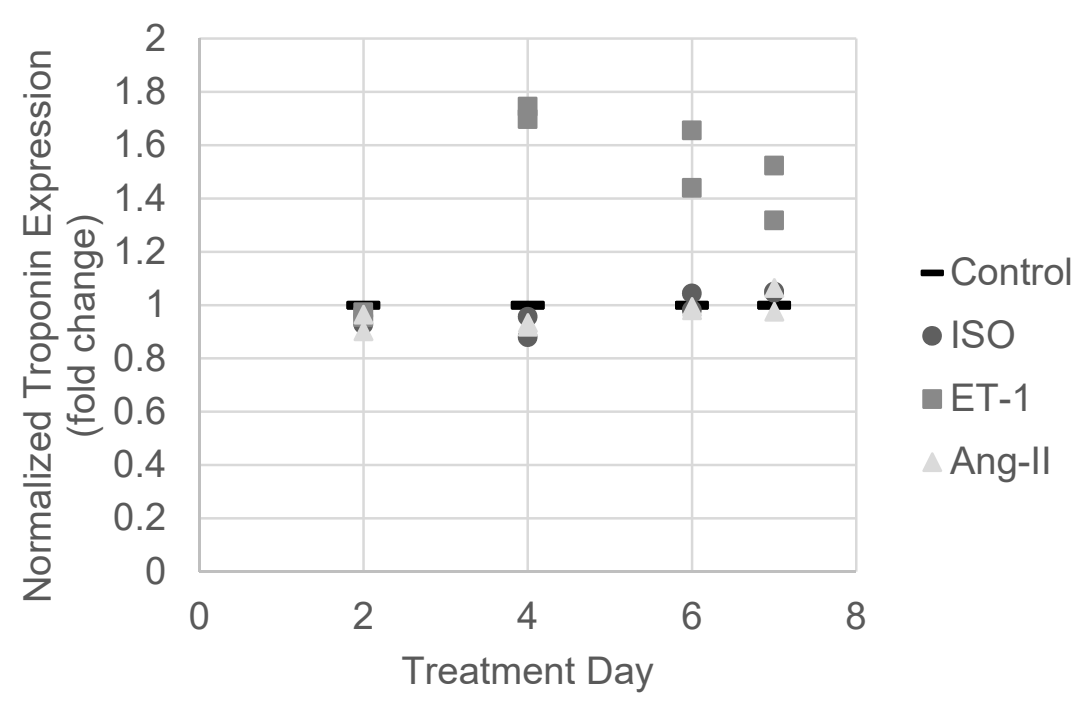

Figure S1. Quantification of soluble human troponin in the culture media of Hes2 untreated biowires (control) or biowires treated with isoproterenol (ISO), angiotensin II (AngII) or endothelin-1 (ET1) for 2, 4, 6 and 7 days. The values measured in the drug treated groups were normalized to the control biowires $(n=2)$. 


\section{Supplemental Figure 2}
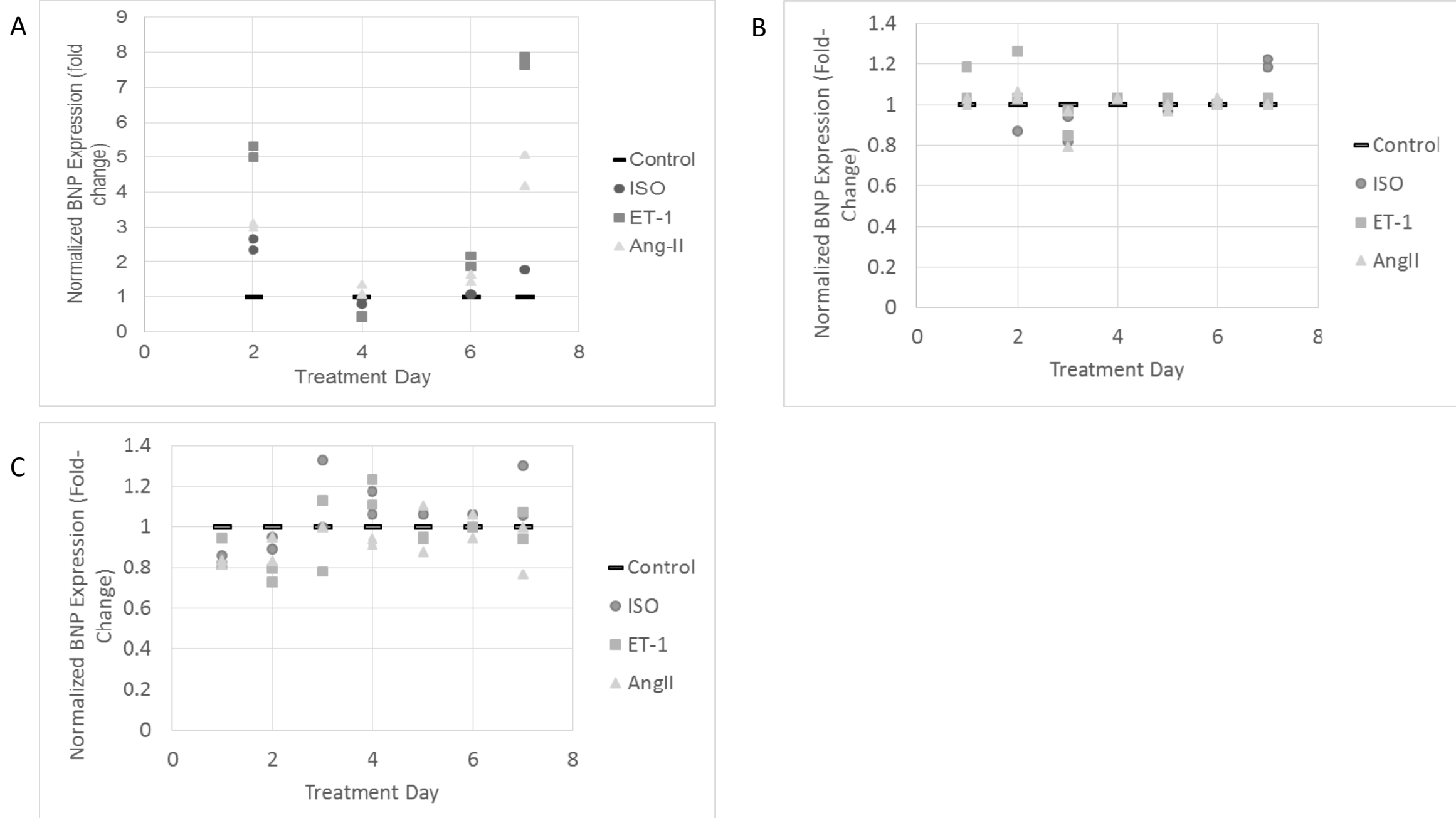

Figure S2. Quantification of soluble BNP in the culture media of Hes2 (A), Hes3 (B) and BJ1D (C) biowires without treatment (control) or treated with isoproterenol (ISO), angiotensin II (AngII) or endothelin-1 (ET-1) on the indicated treatment day. Note that culture media was collected on alternating days for the Hes2 biowires and daily for the BJ1D and Hes3 biowires. Values were normalized to the control biowires ( $n=2$ biowires/well, with $n=2$ wells). 


\section{Supplemental Figure 3}

A

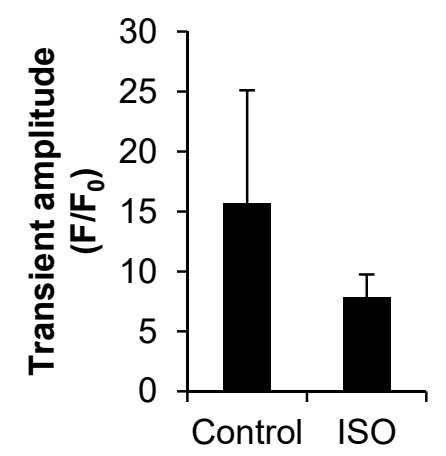

C

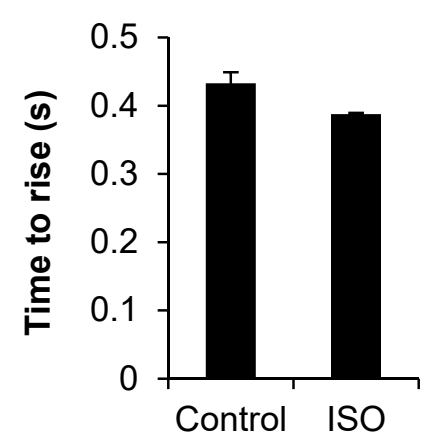

B

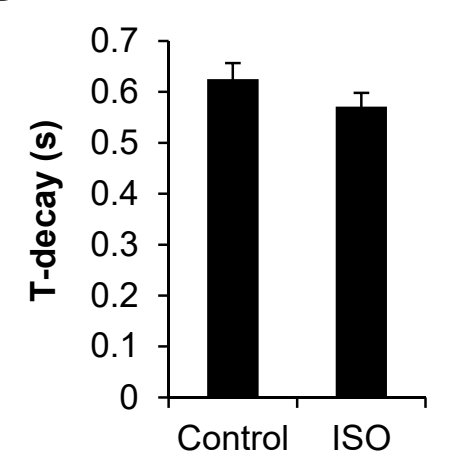

D

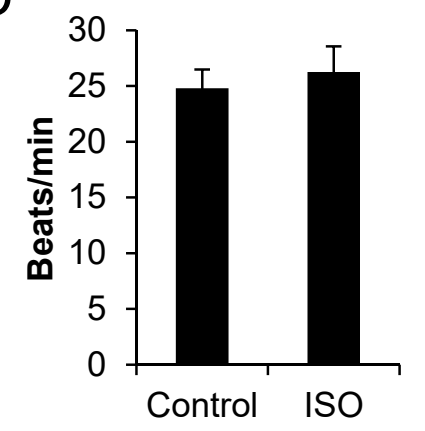

Figure S3. Effect of chronic isoproterenol treatment on calcium transients. There were no significant differences between calcium transient amplitude (A), $\tau$-decay (B) or time to rise (C) in calcium transients of isoproterenol-treated cells vs. non-treated controls with similar beating rates (beats/min) (D). Data are presented as average \pm s.d. 
Table S1: Primer sequences

\begin{tabular}{|c|c|c|}
\hline Gene & Forward (5'-3') & Reverse (3'-5') \\
\hline NPPA & GAACCAGAGGGGAGAGACAGAG & CCCTCAGCTTGCTTTTTAGGAG \\
\hline NPPB & TTCCTGGGAGGTCGTTCCCAC & CATCTTCCTCCCAAAGCAGCC \\
\hline MYH6 & TCAGCTGGAGGCCAAAGTAAAGGA & TTCTTGAGCTCTGAGCACTCGTCT \\
\hline MYH7 & TCGTGCCTGATGACAAACAGGAGT & ATACTCGGTCTCGGCAGTGACTTT \\
\hline GAPDH & GAGTCAACGGATTTGGTCGT & GACAAGCTTCCCGTTCTCAG \\
\hline
\end{tabular}

